\title{
EL SECTOR TERCIARIO EN ESPAÑA \\ ANTES DE 1936: UNA NOTA \\ DE ESCEPTICISMO SOBRE \\ LAS ESTIMACIONES AL USO
}

GABRIEL TORTELLA

Universidad de Alcalá de Henares

Con la excepción de Mulhall y Prados de la Escosura ${ }^{1}$, no se han hecho estimaciones de la «Renta Terciaria» o renta del Sector Servicios para periodos anteriores a 1900. Para la primera mitad del siglo $\mathrm{xx}$, aparte de la ya citada y recientísima de Prados, contamos tan sólo con dos estimaciones, que son parte de las conocidas series de Renta Nacional calculadas por el Consejo de Economía Nacional (CEN) y por Julio Alcaide Inchausti. Hace algún tiempo, con motivo de una larga investigación en equipo (el llamado «Proyecto Europeo», patrocinado por el Banco de España), tuve ocasión de examinar con algún detalle los trabajos donde se explica cómo se llevó a cabo la confección de estas dos series. El resultado de este examen fue la redacción de un informe acerca de los graves problemas de fiabilidad que planteaba el uso de estas estimaciones. El informe circuló entre los miembros del equipo, pero no tuvo ninguna publicidad. La presente nota es una versión abreviada y actualizada de aquel informe. Me han animado a publicarla, de un lado, los compañeros del equipo $y$, de otro, el deseo de poner en guardia a los colegas de los peligros que entraña el depositar excesiva confianza en las tan citadas series.

Es bien sabido que ambas estimaciones, aunque presentan claras diferencias, están basadas en las mismas estadísticas de producción, las recopiladas por el CEN. Examinemos el método seguido en ambos trabajos por lo que respecta al Sector Terciario o de «Servicios»:

1 Las estimaciones de Mulhall, que, como es bien sabido, se refieren a la Renta Nacional, pueden verse, refinadas y sistematizadas, en Prados (1982); la estimación de Prados, específica ya para el sector terciario, y parte de una estimación por tramos de la Renta Nacional española en el siglo xIX y primer tercio del $\mathbf{x x}$, puede verse en Prados (en prensa), cap. 1, esp. cuadro $1-1$. 


\section{La estimación del Consejo de Economia Nacional}

Esta estimación, contenida en el volumen I del libro La Renta Nacional de España por la Comisión de la Renta Nacional, publicado en $1945^{2}$, y comprendiendo el período 1906-1942, se ha elaborado por medios tan toscos que casi provocan incredulidad en un lector atento.

En palabras de la Comisión, el cálculo se ha basado simplemente en el supuesto de que «la producción terciaria [...] es proporcional a la población empleada en este sector» ${ }^{3}$. Pese a lo burdo del supuesto inicial, sin embargo, no queda totalmente claro cómo se ha pasado de esta simple premisa a las cifras efectivas. En la página 229 la Comisión ofrece un cuadro, basado en los Censos, en que se clasifica la población activa por su ocupación en los tres grandes sectores (Primario, Secundario y Terciario) en las fechas de 1900, 1910,1920 y $1930^{4}$. Aparte de no especificar el procedimiento seguido para interpolar estas proporciones para los años no censales, la Comisión tampoco explica cómo se pasa de la proporción de población activa en el Sector Terciario a la proporción de Renta Nacional generada en el Sector Terciario. Es posible que la Comisión se haya limitado a suponer que ambas proporciones fueran idénticas, lo cual equivaldría a afirmar que la productividad del Sector Terciario fuera igual a la productividad media de toda la economía. El supuesto sería altamente discutible. No es seguro, sin embargo, que ése sea el procedimiento seguido por la Comisión.

La explicación que la Comisión da de sus métodos es por demás enigmática ${ }^{5}$. Su única virtud es la extrema brevedad. Por una parte, se nos dice, hay que considerar la población empleada en el Sector Terciario, cuyos datos se ofrecen en el «Anejo $V$ » (se trata del cuadro de la página 229 de que acabo de hablar). $\mathrm{Y}$ a esto agrega: «El otro elemento a considerar era la renta total, que en el plazo considerado la hemos sustituido por la dirección fundamental de la producción.» El significado de esta frase es decididamente esotérico. Aparte de la cláusula «en el plazo considerado», que parece deliberadamente introducida para ofuscar al lector, tenemos dos conceptos de difícil interpre-

2 Véase cita completa en la bibliografía, Consejo de Economía Nacional (1945).

${ }^{3}$ P. 283, dentro del «Anejo V. La población de España».

- En la p. 231 se ofrece un cuadro que muestra la «Distribución [porcentual] de la población activa de varios países», sin más indicación de fechas que la expresión entre paréntesis «Según censos recientes». Para España se dan dos conjuntos de cifras, las calculadas por Colin Clark (1942) y las obtenidas por la Comisión a partir del Censo de 1930. La Comisión no se molesta en informar al lector a qué año se refieren las cifras de Colin Clark. Ambos conjuntos de porcentajes difieren bastante; en concreto, la población ocupada en el Sector Terciario sería el 18,4 por 100, según Colin Clark, y el 27,7 según la Comisión. El lector buscará en vano un comentario sobre tal discrepancia.

${ }^{5}$ Véase «Anejo XI. La producción terciaria y la Renta Nacional», pp. 283-284. 
tación: el de «renta total» y, sobre todo, el de su misteriosa sustituta, la «dirección fundamental de la producción».

Empecemos por el concepto menos difícil, el de «renta total». Es sabido que la Comisión basó sus estimaciones en tres índices: uno de producción agraria, otro de producción industrial y otro de nupcialidad, el último como (muy discutible) indicador de coyuntura. Estos índices los aplicó a las estimaciones de Vandellós y del Banco Urquijo para la Renta Nacional en 1923, con una buena dosis de correcciones y extrapolaciones que ni es fácil desentranar ni es necesario explicar aquí (véanse especialmente el cap. V y el Anejo X). Parece que esa serie resultante de aplicar los índices a las cifras de Renta Nacional de un año base es la llamada «renta total». Pero debemos tener presente que esta serie de «renta total» está construida sin el menor esfuerzo por conocer la verdadera evolución en el Sector Terciario; es decir, se supone que la «Renta Terciaria» es una fracción constante de la «renta total» (la fracción correspondiente al año base); y esta rigidez le parece a la Comisión, con razón, inaceptable. En realidad, la Comisión no manifiesta esto claramente (los redactores del libro parecen considerar la claridad como un vicio a evitar), pero no veo qué otra razón puede haber persuadido a la Comisión a sustituir la «renta total» por la «dirección fundamental de la producción» (aparte del natural deseo de complicar las cosas). Tengamos presente que, de utilizar la «renta total» para calcular la «Renta Terciacia», el CEN estaría incurriendo en un razonamiento circular: estimar la parte a partir del todo, que, a su vez, se ha calculado haciendo un supuesto acerca de la parte.

¿Qué es la «dirección fundamental de la producción»? Unas páginas antes (p. 276) encontramos la respuesta, aunque, por supuesto, muy confusa: la «dirección fundamental [... viene] representada por la recta interpolada entre los datos correspondientes [ ... y además] tiene un paralelismo con la dirección fundamental de las cifras de renta». Mi interpretación de este galimatías es la siguiente. La "dirección fundamental de la producción» es la recta ajustada a los índices de producción ${ }^{6}$, es decir, al índice ponderado de producción agraria e industrial. Y la Comisión afirma, o supone, que la pendiente de esa recta ajustada es muy parecida a la pendiente de la recta ajustada a los. valores, aún desconocidos, de la «renta total». (Por eso, imagino, puede sustituirse una por otra.) Pero todo esto equivaldría a decir que la Renta Nacional crecía a la misma tasa que el índice de producción física (el índice combinado de producción agraria, producción industrial y nupcialidad); es

- En la p. 107 se dice que la «dirección fundamental» del indice de producción viene «expresada por una función lineal del tiempo». Ahora bien, como el índice de producción es una serie temporal, la variable independiente es el tiempo; luego la «dirección fundamentalw parece ser el coeficiente angular de la recta ajustada. 
decir, a seguir afirmando, por confusamente que sea, que el Sector Terciario representaba una fracción constante de la Renta Nacional.

¿Por qué ha de ser ello así? ¿Por qué afirma la Comisión una y otra vez -aunque con justificadas vacilaciones- que el Sector Terciario es una fracción constante de la Renta Nacional? Muy sencillo: porque lo dice Colin Clark. Esto se enuncia nítidamente en la página 71: «existe una ley general para todos los países, demostrada por Colin Clark, en virtud de la cual existe una relación definida entre la renta real por cabeza y la producción terciaria por habitante» " . Y ahora sí que se deja la cosa clara: «la relación encontrada por Colin Clark entre la renta y el volumen de la producción terciaria es una sencilla relación lineal» (p. 283). Sin embargo, por razones que ya no están tan claras, «los datos de que disponíamos no nos han permitido aplicar en toda su pureza el método de aquel insigne estadístico». El método que ha seguido la Comisión del CEN es, sin duda, más rebuscado que el propuesto por el insigne estadístico, pero no parece justificable en modo alguno, aparte de que ni se entiende su desarrollo ni parece aplicable su resultado final. Veamos cómo lo describe la propia Comisión (pp. 283-284):

Entonces, refiriéndonos como punto de origen al año de comienzo de siglo, hemos determinado la siguiente ecuación, en la que $Y$ representa la dirección de la renta y $X$ la dirección fundamental de la producción terciaria; la ecuación resultante es:

$$
Y=11,9+0,995 X
$$

¿Cómo se ha «determinado» la tal ecuación? Misterio absoluto. La interpretación de la ecuación también resulta opaca. ¿Qué significa ese término independiente cercano a 12 ? Yo, desde luego, no lo sé. Lo que sé es que si, como se dice en la página 58 , la «producción total» creció a una tasa media del 2,08 por 100 anual, suponiendo que renta y producción total vengan a ser lo mismo (ya que, según vimos antes, la «dirección fundamental de la producción [...] tiene un paralelismo con la dirección fundamental de las cifras de renta»), resultará que la «dirección [es de suponer que fundamental] de la renta", representada en la ecuación anterior por $Y$, será de 0,0208 , con lo cual el valor de $X$, «dirección fundamental de la producción terciaria», sería de $-11,94$, aproximadamente: un disparate. Pero si, lo que es más ve-

${ }^{\top}$ En la p. 112 se ofrece una nueva versión de la «ley general»: «El estadístico senor Colin Clark ha encontrado que entre la renta real y la producción terciaria existe una definida relación funcional.» Y en la p. 283 se remacha tal afirmación: «hemos empleado la relación funcional demostrada por Colin Clark entre la renta total y el volumen de producción terciaria». 
rosímil, el valor de la «dirección fundamental de la producción terciaria» fuese, por ejemplo, del 1 por 100 anual, el valor de la «dirección de la renta» sería, aproximadamente, de $11,91[Y=11,99+(0,995 \times 0,01)]$ : otro disparate. ¿Habrá una errata en las cifras de la ecuación?

Podríamos intentar rastrear cuál de las posibles fórmulas (la extraña ecuación, el supuesto de una participación constante de la "Renta Terciaria» dentro de la Renta Nacional, el supuesto de una participación de la «Renta Terciaria» igual a la fracción de población activa ocupada dentro del sector, o una combinación de las anteriores) utilizó efectivamente la Comisión del CEN, pero temo que el resultado no compensara el esfuerzo ${ }^{8}$. Para el interesado lo importante y suficiente es saber que la «Renta Terciaria» fue calculada por métodos muy burdos y de manera doblemente indirecta, es decir, considerándola como una función de la producción de los sectores primario y secundario, que ya se había estimado por procedimientos más bien indirectos. En mi opinión, el historiador que quiera utilizar las cifras de Renta Nacional del CEN deberá atenerse con precaución a los índices de producción real (Primaria y Secundaria) y tomar de otra fuente la «Renta Terciaria».

\section{La estimación-corrección de Julio Alcaide}

Esto es en parte lo que trató de hacer Julio Alcaide Inchausti ${ }^{9}$; por desgracia, su «resivión urgente» tampoco parece totalmente satisfactoria; y escribo «parece» de manera deliberada, porque del texto de ese trabajo no se deduce claramente tampoco el método que ha seguido. Hay que señalar, en primer lugar, que las estimaciones de Alcaide son tan indirectas como las del CEN, ya que los datos básicos que utiliza son los de este organismo, limitándose simplemente a mejorar las ponderaciones de los índices. Las únicas informaciones nuevas que introduce Alcaide en sus estimaciones son la demográfica y la de precios, campos ambos en los que ha contado con aportaciones del Instituto Nacional de Estadística publicadas posteriormente a la conclusión del trabajo del CEN.

En cuanto a su estimación de la «Renta Terciaria», Alcaide critica severàmente al CEN por cuanto

al prescindir del comportamiento del sector terciario [...] cometió un acusado error, ya que es obvio el factor de equilibrio que en

- La mayor dificultad radicaría en que la Comisión no ofrece explícitamente ninguna serie de «Renta Terciaria»; se diria que le ha faltado valor.

- Alcaide (1976). 
la evolución de la Renta Nacional compete a las actividades de servicios, no necesariamente ligadas a la evolución de la producción material ${ }^{10}$.

Si bien Alcaide tiene toda la razón en afirmar que el CEN «cometió un acusado error» al tratar tan de pasada al Sector Terciario, la segunda parte de la cita parece contener el germen de un principio peligroso: el de utilizar la «Renta Terciaria» como «factor de equilibrio» que le permita a Alcaide eliminar las fluctuaciones indeseadas de la serie de producción real obtenida de los índices del CEN. Sin duda, es correcta su afirmación de que la «Renta Terciaria» no tiene por qué estar muy estrechamente ligada a la producción material, por cuanto al menos una parte de la «Renta Terciaria», la generada por la Administración, la Sanidad o la Educación de un determinado año, por ejemplo, no tiene por qué estar relacionada por un simple coeficiente con la producción física de ese mismo año. Otra fracción, la generada por los sectores de Comercio, de Transportes o de Crédito, por ejemplo, sí estará estrechamente relacionada con el volumen de producción de los sectores reales ${ }^{11}$. Sin embargo, eso no significa que se deba o pueda considerar a esa fracción «independiente» del Sector Terciario como un «factor de equilibrio»: en la medida en que esas actividades están financiadas por el Sector Público ( $\mathrm{y}$, sin duda, se trata de una medida considerable), su volumen dependerá de la política, no solamente económica, del Gobierno (que puede ser, o no, anticíclica) y del volumen de renta de años anteriores, además de depender del volumen de renta del año corriente; también estarán relacionadas con variables demográficas y monetarias. Nada de todo esto, excepto una política económica deliberadamente anticíclica del Gobierno, altamente improbable antes de Keynes, hace suponer que esa fracción de «Renta Terciaria» vaya a actuar como «factor de equilibriom. Por todo ello, esta expresión de Alcaide me hace temer que sus estimaciones de la «Renta Terciaria» estén arregladas de manera que corrijan las que su autor considere como aberraciones de sus estimaciones de producción física obtenidas a partir de los índices del CEN.

El problema reside en que Alcaide no deja claro cuál ha sido su método estimativo. Lo más explícito que he encontrado en su texto es lo siguiente:

Para completar la información cuantitativa de producción se ha estimado un índice de servicios en base a la evolución del empleo terciario y adaptado a los cambios en la producción total. Sobre una producción para el año 1906 del 40 por 100 en agri-

10 Alcaide (1976), p. 1128.

"Véase O'Brien (1983). 
cultura, 25 por 100 en industria y 35 por 100 en servicios, se ha pasado al cálculo de un índice general de producción, índice que suaviza las profundas alteraciones del índice calculado por el CEN ${ }^{12}$.

Es difícil saber qué significa esto con exactitud. Por ejemplo, ¿qué son las «alteraciones» de un índice? ¿Cómo precisamente «se ha estimado un índice de servicios en base a la evolución del empleo terciario y adaptado a los cambios en la producción total»? La palabra «productividad» no se formula en toda esta explicación, pero es crucial para comprender lo que ha tratado de hacer Alcaide, puesto que él mismo nos dice que las dos bases de su estimación son la población activa y el índice de producción, las dos componentes de un índice de productividad.

Alcaide nos ofrece dos tablas (la número 4 y la número 8 ), sin embargo, donde se dan estimaciones de productividad. En la tabla 8 se ve claramente que la productividad del Sector Terciario, según esta estimación, era mucho más alta que la de los otros grandes sectores. Lo mismo se desprende de la tabla 4 , donde también se dan cifras de valor añadido por persona ocupada en cada sector. ¿Qué relación hay entre una y otra tabla? A primera vista pudiera pensarse que la número 4 nos da, como dice su encabezamiento, el "Valor añadido bruto por persona activa», en pesetas corrientes, y que la número 8 , cuyo encabezamiento reza en parte «Valor añadido por persona activa», con un paréntesis que dice «En términos de renta bruta, a pesetas constantes de 1964», nos da la misma magnitud de la tabla 4 en términos reales, es decir, en pesetas constantes. Sin embargo, no es así: como muestra el cuadro A adjunto, esto parece ser así hasta 1924; pero a partir de los valores de ese año, las relaciones entre las productividades sectoriales y la media o total no son iguales (tampoco lo son el año 1922).

Aunque las diferencias entre una y otra tabla evidenciadas en el cuadro A, cuando las hay, son pequeñas, hay dos cuestiones muy sorprendentes. En primer lugar, que ambas tablas coincidan para los años anteriotes a 1924 menos uno y ya no vuelvan a coincidir para los años posteriores, ya que si coinciden es que, como parece lógico, son la misma serie ( flactada en el otro), y si no coinciden es que no son la misma serie. Pero que coincidan en un tramo y no en el otro parece reflejar un extraño caso de doble personalidad serial. $Y$ en segundo lugar que, cuando hay divergencias, todas las productividades seriales de la tabla 8 sean más altas con relación a la media que las de la tabla 4. Según el propio Alcaide, la diferencia entre las tablas reside en que la número 8 se ha calculado *deflactando la renta

${ }^{12}$ Alcaide (1976), p. 1128. 


\section{CUADRO A}

Productividades sectoriales según las tablas 4 y 8 de la "Revisión Urgente» de Alcaide

(En porcentajes sobre la Productividad Media o Total Nacional)

\begin{tabular}{|c|c|c|c|c|c|c|}
\hline \multirow[b]{2}{*}{ Años } & \multicolumn{2}{|c|}{$\begin{array}{c}\text { (1) } \\
\text { Agricultura y Pesca }\end{array}$} & \multicolumn{2}{|c|}{$\begin{array}{c}\text { (2) } \\
\text { Industria }\end{array}$} & \multicolumn{2}{|c|}{$\begin{array}{l}\text { (3) } \\
\text { Servicios }\end{array}$} \\
\hline & $A$ & $B$ & $A$ & B & $A$ & $B$ \\
\hline 1901 * & 69,5 & 69,5 & 128,0 & 128,0 & 189,0 & 189,0 \\
\hline 1905 * & 70,4 & 70,4 & 125,1 & 125,1 & 182,9 & 182,9 \\
\hline 1910 * & 70,1 & 70,1 & 123,5 & 123,5 & 179,1 & 179,1 \\
\hline 1915 * & 72,3 & 72,3 & 123,5 & 123,5 & 179,1 & 179,1 \\
\hline $\begin{array}{l}1920 \text { * } \\
1921 \text { * } \\
1922 \\
1923 \text { * } \\
1924 \text { * }\end{array}$ & $\begin{array}{l}71,3 \\
70,6 \\
70,8 \\
72,6 \\
74,8\end{array}$ & $\begin{array}{l}71,3 \\
70,6 \\
72,8 \\
72,6 \\
74,8\end{array}$ & $\begin{array}{l}111,3 \\
121,1 \\
113,8 \\
114,7 \\
109,7\end{array}$ & $\begin{array}{l}111,3 \\
121,1 \\
117,0 \\
114,7 \\
109,7\end{array}$ & $\begin{array}{l}170,1 \\
159,2 \\
163,5 \\
155,2 \\
153,1\end{array}$ & $\begin{array}{l}170,1 \\
159,2 \\
168,0 \\
155,2 \\
153,1\end{array}$ \\
\hline $\begin{array}{l}1925 \\
1926 \\
1927 \\
1928 \\
1929\end{array}$ & $\begin{array}{l}77,1 \\
77,7 \\
79,4 \\
78,9 \\
80,0\end{array}$ & $\begin{array}{l}77,5 \\
78,6 \\
80,3 \\
80,3 \\
81,9\end{array}$ & $\begin{array}{r}103,2 \\
100,6 \\
98,1 \\
95,2 \\
97,5\end{array}$ & $\begin{array}{r}103,8 \\
101,8 \\
99,2 \\
96,9 \\
99,8\end{array}$ & $\begin{array}{l}153,2 \\
153,5 \\
151,0 \\
154,9 \\
148,5\end{array}$ & $\begin{array}{l}154,0 \\
155,2 \\
152,7 \\
157,7 \\
152,1\end{array}$ \\
\hline $\begin{array}{l}1930 \\
1931 \\
1932 \\
1933 \\
1934\end{array}$ & $\begin{array}{l}73,2 \\
74,2 \\
80,9 \\
79,8 \\
82,5\end{array}$ & $\begin{array}{l}76,0 \\
77,1 \\
84,1 \\
83,5 \\
86,8\end{array}$ & $\begin{array}{r}100,0 \\
98,8 \\
96,2 \\
94,8 \\
93,7\end{array}$ & $\begin{array}{r}103,9 \\
102,7 \\
100,0 \\
99,3 \\
98,6\end{array}$ & $\begin{array}{l}158,4 \\
157,1 \\
145,5 \\
149,0 \\
144,1\end{array}$ & $\begin{array}{l}164,7 \\
163,4 \\
151,3 \\
156,0 \\
151,6\end{array}$ \\
\hline 1935 & 77,4 & 81,6 & 97,3 & 102,5 & 148,2 & 156,2 \\
\hline
\end{tabular}

* Año en que las relaciones entre las productividades sectoriales y la productividad media total son iguales en ambas tablas.

FUENTES:

Cols. A: Tabla 4, p. 1140 , en Alcaide (1975).

Cols. B: Calculado a partir de tabla 8 , p. 1148 , en ibidem. 
bruta por persona activa por el índice general de precios, no por los precios específicos de cada sector, lo que permite contemplar la evolución real de la renta por persona activa de cada sector productivo» (p. 1133). No entiendo por qué esto produce una coincidencia en los primeros años y una divergencia en los últimos, ni por qué produce un alza general relativa en los tres componentes sectoriales del valor añadido total. Sospecho, incluso, que esto no es lógico ni matemáticamente posible; en mi opinión, por tanto, y mientras no se demuestre lo contrario, las series de productividad y de «Renta Terciaria» de Alcaide merecen muy poca confianza.

A todo esto, por supuesto, ignoramos si las series de productividad en las tablas 4 y 8 han servido para calcular la «Renta Terciaria» o si, por el contrario, es la serie de «Renta Terciaria» contenida en la tabla 3 la que ha servido para calcular las series de productividad. El texto citado anteriormente (el de la p. 1128 de Alcaide) me parece sugerir la segunda posibilidad como más probable. En todo caso, quien esto escribe sigue ignorando, después de leer y releer una y otra vez la «Revisión urgente», qué fuentes y qué métodos ha utilizado Alcaide para calcular la renta del Sector Terciario.

¿Qué consecuencia pueden tener estos métodos irregulares de estimación? $\mathrm{O}$, dicho de otra manera, ¿cuán mala o errónea es la serie de «Renta Terciaria» de Alcaide? ${ }^{13}$. El cuadro B ofrece una contrastación con una serie mucho más fiable, la recientemente calculada por Prados de la Escosura. Aunque los detalles del cálculo de Prados tampoco se conocen, sus fuentes si ${ }^{14}$. Se trata de un indice basado en series de producción de los principales subsectores del Terciario: administración pública, banca, comercio, renta de edificios y viviendas, transporte, comunicaciones, profesiones liberales y servicio doméstico. Los cinco primeros índices se han construido a partir de investigaciones recientes, los tres últimos a partir de estadísticas oficiales y censales, y de las conocidas estimaciones de Vandellós. Al cabo, Prados nos ofrece un índice de producto para una serie de años terminados en cero, de 1800 a 1930 .

El cuadro B contiene una comparación, para tres puntos temporales en el siglo xx (1910, 1920 y 1930), entre los índices de Prados y unos índices construidos por mí enteramente basados en los datos de Alcaide. La principal conclusión que se deduce de esta comparación es que las cifras de Alcaide parecen subestimar gravemente la «Renta Terciaria», hasta el extremo que, según mis cálculos basados en sus datos ( $\mathrm{mi}$ sencillo método se especifica claramente en el cuadro), la productividad del sector habría descendido (o, cuando más, se habría estancado) entre 1910 y 1930 de ser buena la serie de

${ }^{13}$ Recordemos que la del CEN no se puede verdaderamente someter a contrastación porque no ha sido publicada como tal serie.

${ }_{14}$ Prados (en prensa), cap. 1. 


\section{CUADRO B}

Comparación de los indices de «Renta Terciaria» de Alcaide y de Prados

(1)

\begin{tabular}{|c|c|c|}
\hline \multirow[b]{2}{*}{ Años } & \multicolumn{2}{|c|}{ Producto } \\
\hline & $A$ & $P$ \\
\hline 1910 & 100,0 & 100,0 \\
\hline 1920 & 122,5 & 125,3 \\
\hline 1930 & 152,0 & 178,1 \\
\hline
\end{tabular}

(2)

\begin{tabular}{|c|c|}
\hline$A$ & $P$ \\
\hline 100,0 & 100,0 \\
\hline 114,6 & 117,3 \\
\hline 128,8 & 150,6 \\
\hline
\end{tabular}

(3) Producto por trabajador

\begin{tabular}{rrr}
\hline \multicolumn{1}{c}{$A$} & & $P$ \\
100,0 & & 100,0 \\
94,4 & & 102,8 \\
99,8 & & 115,8
\end{tabular}

Furantrs Y MÉTODO:

Cols. A: Calculado a partir de Alcaide (1976). La $*$ Renta Terciaria» proviene de la tabla 3. He atribuido a los años 1910,1920 y 1930 los valores de las medias trienales centradas en esos años, y he deflactado esos valores con el «Indice de precios implícitos PNB» de la tabla 6. Las cifras de Población Total y de Población Activa en el Sector de Servicios provienen de las tablas 1 y 2.

Cols. P: Prados (en prensa), cuadro 1-1.

Alcaide, mientras que, según Prados, habría aumentado en un 16 por 100 en ese mismo intervalo. Las discrepancias que muestra el cuadro B son elocuentes y a todas luces excesivas. En especial, la serie de Alcaide parece no reflejar suficientemente el indudable y general crecimiento de los años veinte.

En resumidas cuentas, muy poco o nada me parece aprovechable en las estimaciones al uso de la «Renta Terciaria», lo cual vicia en medida considerable los valores de Renta Nacional de esas estimaciones. Evidentemente, no estoy sugiriendo que lancemos por la borda el trabajo del CEN y las correcciones de Alcaide, pero sí que reconozcamos sus serias deficiencias, que los utilicemos con gran cautela y que consideremos como prioritaria la tarea de mejorar nuestro conocimiento de las series macroeconómicas. 


\section{BIBLIOGRAFIA CITADA}

Alcaide Inch austi, Julio (1976): «Una revisión urgente de la serie de Renta Nacional española en el siglo XX», en Instituto de Estudios Fiscales (1976), pp. 1127-1150.

Clark, Colin (1942): The Economics of 1960, Londres: MacMillan.

Consejo de Economía Nacional (1945): La Renta Nacional de España por la Comisión de Renta Nacional, vol. I: Planteamiento general del problema. Estimaciones indirectas y primeras cifras, Madrid: Estado español.

Instituto DE Estudios Fiscales (1976): Datos básicos para la Historia financiera de España (1850-1975), vol. I, Madrid: Ministerio de Hacienda.

Fremdling, Rainer, y Patrick K. O'BrIEN (1983): Productivity in the Economies of Europe, Stuttgart: Klett-Cotta.

O'BrIEN, Patrick K. (1983): «The Analysis and Measurement of the Service Economy in European Economic History», en Fremdling y O'Brien (1983), pp. 79-89.

PRAdos de la Escosura, Leandro (1982): Comercio exterior y crecimiento económico en España, 1826-1913: tendencias a largo plazo, Madrid: Banco de España, Servicio de Estudios (Estudios de Historia Económica, núm. 7).

- (en prensa): De Imperio a nación. Crecimiento y atraso económico en España (17801930). 